(2) Open Access Full Text Article

\title{
Experimental study of tissue-engineered cartilage allograft with RNAi chondrocytes in vivo
}

This article was published in the following Dove Press journal:

Therapeutics and Clinical Risk Management

8 May 2014

Number of times this article has been viewed

\author{
Zhenghui Wang' \\ Xiaoli $\mathrm{Li}^{2}$ \\ Xi-Jing $\mathrm{He}^{3}$ \\ Xianghong Zhang' \\ Zhuangqun Yang ${ }^{4}$ \\ Min $\mathrm{Xu}{ }^{\prime}$ \\ Baojun $\mathrm{Wu}^{\prime}$ \\ Junbo $\mathrm{Tu}^{5}$ \\ Huanan Luo' \\ Jing Yan'
}

'Department of Otolaryngology Head and Neck Surgery, ${ }^{2}$ Department of Dermatology, ${ }^{3}$ Department of Orthopedics, The Second Hospital, Xi'an Jiaotong University, ${ }^{4}$ Department of Plastic and Burns Surgery, The First Hospital, Xi'an Jiaotong University, ${ }^{5}$ Department of Oral and Maxillofacial Plastic Surgery, The Stomatological Hospital, Xi'an Jiaotong University, Xi'an, People's Republic of China
Correspondence: Baojun Wu; Min Xu Department of Otolaryngology Head and Neck Surgery, The Second Hospital, Xi'an Jiaotong University, Xi Wu Road, Xi'an 7I 0004, People's Republic of China

Email wubaojun bone@l63.com; ent551205@163.com
Purpose: To determine the effects of RNA interference (RNAi) on chondrocyte proliferation, function, and immunological rejection after allogenic tissue-engineered cartilage transplantation within bone matrix gelatin scaffolds.

Methods: Seven million rat normal and RNAi chondrocytes were harvested and separately composited with fibrin glue to make the cell suspension, and then transplanted subcutaneously into the back of Sprague Dawley rats after being cultured for 10 days in vitro. Untransplanted animals served as the control group. The allograft and immunological response were examined at 1, 2, 4, 8, and 12 months postoperatively with hematoxylin and eosin histochemical staining, immunohistochemical staining (aggrecan, type II collagen, class I and II major histocompatibility complex), and flow cytometry for peripheral blood cluster of differentiation $4^{+}\left(\mathrm{CD}^{+}\right)$ and CD8 ${ }^{+}$T-cells.

Results: There was no infection or death in the rats except one, which died in the first week. Compared to the control group, the RNAi group had fewer eukomonocytes infiltrated, which were only distributed around the graft. The ratio of $\mathrm{CD}^{+} / \mathrm{CD}^{+} \mathrm{T}$-cells in the RNAi group was significantly lower than the normal one $(P<0.05)$. There were many more positively stained chondrocytes and positively stained areas around the cells in the RNAi group, which were not found in the control group.

Conclusion: The aggrecanase- 1 and aggrecanase-2 RNAi for chondrocytes decreased the immunological rejection effect.

Keywords: chondrocytes, tissue-engineered cartilage, aggrecan, aggrecanase, allograft, immunological response, RNA interference

\section{Introduction}

Cartilage lacks an intrinsic capacity to repair itself when damaged due to cartilage having no nutrition supply (eg, arterial blood supply, venous and lymphatic drainage). Therefore, cartilage transplantation has become one potential method for the reconstruction of cartilage defects. Autogenous or allogenic cartilage and chondrocyte transplantation have been preliminarily employed in clinic applications; however, the disadvantages include limitation of donor material, immune rejection, and low quality of repaired cartilage.

With the development of tissue engineering, tissue-engineered cartilage transplantation has become the most important method to repair damaged cartilage. Appropriate seed cell selection is one of the decisive elements for the survival of grafts in vivo. In recent years, many studies have been performed in this field. ${ }^{1}$ However, degeneration of the graft was commonly observed 2-3 weeks after allogeneic chondrocytes 
transplantation, which was explained by the immune rejection response such as cell-mediated cytotoxicity. ${ }^{2}$ Cartilage is composed of chondrocytes (cartilage cells) embedded in a highly specialized extracellular matrix (ECM). Chondrocytes constitutively express class I and, in some species, class II major histocompatibility complex (MHC). This matrix composed of water ( $80 \%)$, collagen, and proteoglycans - does not allow permeability of molecules greater than $60 \mathrm{kDa}$, and can protect chondrocytes from acceptor antibodies. Therefore, ECM acts as an immunological barrier and makes cartilage a poor "antigen tissue".

ECM is found to be degraded after cartilage transplantation surgery, especially in the first month. ${ }^{3}$ In addition, the degradation of aggrecan was considered an early event in the destruction of articular cartilage. ${ }^{4}$ The key catabolic enzymes for aggrecan are aggrecanase-1 and aggrecanase-2, with specificity effectiveness. In the authors' previous study, the expression of chondrocyte aggrecanase-1 and aggrecanase-2 was silenced by RNA interference (RNAi) technology, leading to the protection of aggrecan from degradation, without interfering with chondrocytic gene phenotype recovery. ${ }^{5}$

In the present study, the authors attempted to reconstruct tissue-engineered cartilage with normal or RNAi-treated allogenic chondrocytes and then transplant them subcutaneously into the back of Sprague Dawley (SD) rats in order to study the effects of aggrecanase silencing the growth of allograft cartilage in vivo, as well as the potential changes in immunological rejective reaction.

\section{Materials and methods Materials and reagents}

The study was approved by the ethics committee of animal research at Xi'an Jiao Tong University (Xi'an, People's Republic of China), and all procedures were carefully conducted following their guidelines of animal research.

$\mathrm{SD}$ rats were obtained from the animal center of Xi' an Jiao Tong University. The short hairpin RNA targeting green fluorescence protein (pshRNA-GFP) lentivector kit was purchased from KangChen Bio-tech Inc. (Shanghai, People's Republic of China). Dulbecco's Modified Eagle's Medium (DMEM), Lipofectamine ${ }^{\circledR} 2000$, and TRIzol ${ }^{\circledR}$ were from Invitrogen (Carlsbad, CA, USA). Fetal bovine serum was from Biological Industries (Kibbutz Beit-Haemek, Israel). Interleukin-1 $\beta$ was from Reprotech Ltd (Rehovot, Israel). The reverse first-strand complementary DNA synthesis kit was from Thermo Fisher Scientific (Waltham, MA, USA). The mouse polyclonal antibody to $\beta$-actin (rat) and the goat antibody to aggrecan (rat) were from Santa
Cruz Biotechnology (Santa Cruz, CA, USA). The aggrecan specific NITEGE373 neoepitope rabbit polyclonal antibody (rat) was from IBEX Technologies Inc. (Montreal, QC, Canada). The mouse polyclonal antibody against collagen II (rat) was from Lab Vision (Fremont, CA, USA). Goat antimouse immunoglobulin $\mathrm{G}$ ( $\mathrm{IgG})$ /horseradish peroxidase, goat antirabbit $\mathrm{IgG} /$ horseradish peroxidase, and rabbit antigoat IgG/horseradish peroxidase were from Zhongshan Biotechnology, Inc. (Beijing, People's Republic of China). The SuperSignal $^{\mathrm{TM}}$ West Pico chemiluminescent kit was from Thermo Fisher Scientific.

\section{Isolation and culture of rat costochondral chondrocytes}

SD rat costal cartilages were isolated and unwrapped from the bone and perichondrium and then diced into fragments of appropriately $1 \mathrm{~mm}^{3}$. Then the cartilages were digested enzymatically with $0.05 \%$ hyaluronidase (Sigma-Aldrich, St Louis, MO, USA) for 0.5 hours at $37^{\circ} \mathrm{C}, 0.25 \%$ trypsin (Gibco, Carlsbad, CA, USA) for 0.5 hours at $37^{\circ} \mathrm{C}$, and subsequently $0.2 \%$ collagenase II (Sigma-Aldrich) overnight at $37^{\circ} \mathrm{C}$ with moderate agitation. After digestion, the number of vital cells was determined by a hemocytometer using trypan blue.

The primary chondrocytes were cultured in culture flasks with high-glucose DMEM $(584 \mathrm{mg} / \mathrm{L}$ glutamine, $50 \mathrm{mg} / \mathrm{L}$ L-ascorbic acid, $5 \times 10^{4} \mathrm{U} / \mathrm{L}$ penicillin, $50 \mathrm{mg} / \mathrm{L}$ streptomycin sulfate, $10 \mathrm{mmol} / \mathrm{L}$ hydroxyethyl piperazineethanesulfonic acid, and $10 \%$ fetal bovine serum) under standard conditions at a final density of $1 \times 10^{5} \mathrm{cells} / \mathrm{cm}^{2}$ as the monolayer. The medium was renewed every second day. The chondrocytes were passage cultured, and the first passage cells were harvested for detection of chondrocyte biological characteristics and used for the following experiment.

\section{RNAi treatment of chondrocytes}

The first passage chondrocytes were seeded on six-well culture plates $\left(1 \times 10^{6}\right.$ cells per well) and cultured. After 1 day, the cells were transfected with the aggrecanase- 1 and aggrecanase-2 plasmids expressing short hairpin RNA using the RNAi technology as previously described. ${ }^{5}$ The RNAi-treated cells were continually cultured and used for the following experiment.

\section{Construction of tissue-engineered cartilage in vitro}

The scaffolds of cancellous bone matrix gelatin (BMG) in a cube shape were supplied by the Fourth Military 
Medical University (Xi'an, People's Republic of China) at a size of $5 \times 5 \times 5 \mathrm{~mm}^{3}$. These scaffolds were sterilized by radiation, and soaked in DMEM for 5 minutes. After being moisture blotted, they were drenched with thrombin solution, then dried and placed into each well of a 96-well culture plate for pre-emergency.

The first passage cells and RNAi-treated cells were detached enzymatically using $0.25 \%$ trypsin and $0.05 \%$ ethylenediaminetetraacetic acid, and suspensed with DMEM containing $20 \%$ fetal calf serum. Then the cells were separately mixed with fibrin glue for cell suspension at a final density of $1 \times 10^{7}$ cells $/ \mathrm{mL}$.

The control and RNAi-treated cells in suspension solution were separately seeded on the prepared BMG scaffold in 96-well plates by the drop technique under sterile conditions ( $50 \mu \mathrm{L}$ suspension per scaffold; $1 \times 10^{7}$ cells $/ \mathrm{mL}$ ), and $200 \mu \mathrm{L}$ DMEM was added into each well after the suspension had completely soaked into the scaffolds. The scaffolds were cultured for 10 minutes in standard conditions for the fibrin glue to solidify and fix to the cells. They were then transferred into the wells of 24-well plates (one scaffold per cell), and $2 \mathrm{~mL}$ DMEM was added to each well for up to 10 days in culture. Half of the culture medium was changed every day. Some scaffolds were removed for cell adhesion, growth, and assay to compare the biological characteristics of the chondrocytes through histological, immunohistochemical staining, and reverse transcription polymerase chain reaction (RT-PCR) after 7 days in culture. The others were continually cultured for the in vivo experiment.

\section{Allotransplantation of tissue-engineered cartilage in vivo}

The female SD rats $(n=85 ; 200 \pm 5 \mathrm{~g})$ were anesthetized with a peritoneal injection of $10 \%$ chloral hydrate solution $(0.35-0.40 \mathrm{~mL} / 100 \mathrm{~g})$ as the transplant acceptor (six animals in each group/time point). The cell-scaffold mixtures were removed and transplanted subcutaneously into the back of SD rats after being cultured for 10 days in vitro using aseptic surgical techniques. The simple scaffold was implanted as a negative control. The SD rat costal cartilage was completely separated, freed from its perichondrium, and implanted subcutaneously into the same batch of SD rats. Otherwise the normal rats were bred as a blank control (ie, non-treated). All animals were bred in the same conditions. The allograft and immunological responses were both observed at postoperative 1, 2, 4, 8, and 12 months through hematoxylin and eosin (HE) histochemical staining, immunohistochemical staining (aggrecan, type II collagen, MHC I, and MHC II), and peripheral blood cluster of differentiation $4^{+}\left(\mathrm{CD}^{+}\right)$, $\mathrm{CD}^{+} \mathrm{T}$-cells by flow cytometry. The biological characteristics and immunological response of allogenic tissueengineered cartilage were evaluated.

\section{Detection of cartilage graft biological characteristics}

For histology and immunohistochemistry, the cartilage grafts were fixed in 4\% paraformaldehyde, dehydrated with a series of graded alcohol, embedded in paraffin, and $6 \mu \mathrm{m}$ thick sections were cut. The sections were stained with HE for morphology and lympholeukocyte infiltration analysis and toluidine blue for sulfated glycosaminoglycans. Immunohistochemistry was performed for aggrecan and collagen II using primary, polyclonal anti-rabbit antibodies (1:100; Santa Cruz). Sections were deparaffinized, digested with methanol and $0.1 \%$ Triton X-100 for membrane permeability, and washed with phosphate buffered saline (PBS). Then, the sections were treated with $3 \%$ hydrogen peroxide at $37^{\circ} \mathrm{C}$ for 30 minutes to inactivate the endogenous peroxidases, incubated with normal goat serum for 30 minutes to block nonspecific binding, and incubated with 1:200 mouse anti-rat collagen II or goat anti-rat aggrecan primary antibody overnight at $4^{\circ} \mathrm{C}$. After washing in PBS, the sections were incubated with horseradish-conjugated anti-mouse or antigoat IgG secondary antibodies for 30 minutes at room temperature. The signal was developed using 3, 3'-diaminobenzidine (DAB kit; Zhongshan Biotechnology Inc., Beiijng, People's Republic of China). The negative control was performed without incubation of the primary antibody.

\section{Detection of the immunological rejection}

The local immunologic reaction was detected by HE staining and immunohistochemistry (MHC I and MHC II) of the grafts with the same methods described above.

The ratio of $\mathrm{CD}^{+}$and $\mathrm{CD} 8^{+}$T-cells in peripheral blood was examined by flow cytometry to estimate the general immunologic reaction. The blood was drawn from the heart of the recipient after the rats were anesthetized with a peritoneal injection, and treated with ethylenediaminetetraacetic acid diluted 1:1 in PBS for anticoagulation. Then, $100 \mu \mathrm{L}$ anticoagulated blood was separately removed to $2 \mathrm{~mL}$ centrifuge tubes, stained with $\mathrm{CD}^{+}$fluorescein isothiocyanate monoclonal antibody (1:100, Santa Cruz) and $\mathrm{CD}^{+}$phycoerythrin monoclonal antibody (1:100, Santa Cruz) for 30 minutes, and then treated with akaryocyte lysate for 10 minutes. The procedures were all performed with protection from light at $4^{\circ} \mathrm{C}$. Then, the cells were washed twice in PBS, fixed in $0.5 \%$ 
paraformaldehyde, and subjected to flow cytometry to detect the percentage of $\mathrm{CD} 4^{+}$and $\mathrm{CD} 8^{+}$lymphocyte subpopulations as previously described. ${ }^{6}$

\section{RT-PCR and cytokine examination}

A TRIzol-based method was used to extract the messenger RNA and then the samples for RT-PCR as previously described (primer, condition, procedures). ${ }^{5}$

For serum cytokine examination, enzyme-linked immunosorbent assay kits (Invitrogen) were used, and the tests were performed as described in the brochure. ${ }^{5}$ Finally, the results were read with a plate reader and calculated to get the final concentration.

\section{Statistical analysis}

Statistical significance of differences in ratios of $\mathrm{CD}^{+}$and $\mathrm{CD} 8^{+}$lymphocyte subpopulations between groups was analyzed using analysis of interclass variance (ANOVA). The software was SPSS 17.0 (SPSS Statistics, Chicago, IL, USA). Differences were considered statistically significant when $P<0.05$.

\section{Results}

\section{The construction of tissue-engineered} cartilage in vitro

HE staining showed chondrocytes entered the mesh of the $\mathrm{BMG}$ and showed proliferation after 7 days in culture. There was a layer of chondrocytes around the BMG. Toluidine blue staining showed that there were positively stained cells in the material. Immunostaining of type II collagen was also observed and there was no significant difference between the two groups. However, the RNAi group showed increased aggrecan staining, suggesting aggregation of proteoglycan in the material.

\section{Transplantation of tissue-engineered cartilage in vivo}

One week after transplantation, there was a layer of fibrous tissue encapsulation around the graft with many lymphocytes in it and a thickening trend over time. The graft could be seen after fibrous tissue encapsulation cut open. The costal cartilage bar kept its original shape post operation, with no obvious absorption.

The stent group began to degrade at 8 weeks, and the volume decreased. The RNAi group retained its original volume at 12 weeks, and displayed the cartilage-like tissue structure. However, the normal chondrocyte group appeared to be absorbed at 8 weeks, the volume obviously reduced, and the texture became crisp at 12 weeks. HE staining showed that the marginal integrity of the graft was maintained and few leukomonocytes were seen in the costal cartilage bar group. There was no inflammatory cell infiltration in the stent group, and the graft construction became raritas and began to degrade at 8 weeks.

In the RNAi and normal chondrocyte groups, there was infiltration of inflammatory cells with lymphocyte predominance around the graft. The cells were fewer in number with no significant increase in the RNAi group, and the chondrocytes showed mass propagation. However, the normal chondrocyte group displayed an opposite trend, ie,
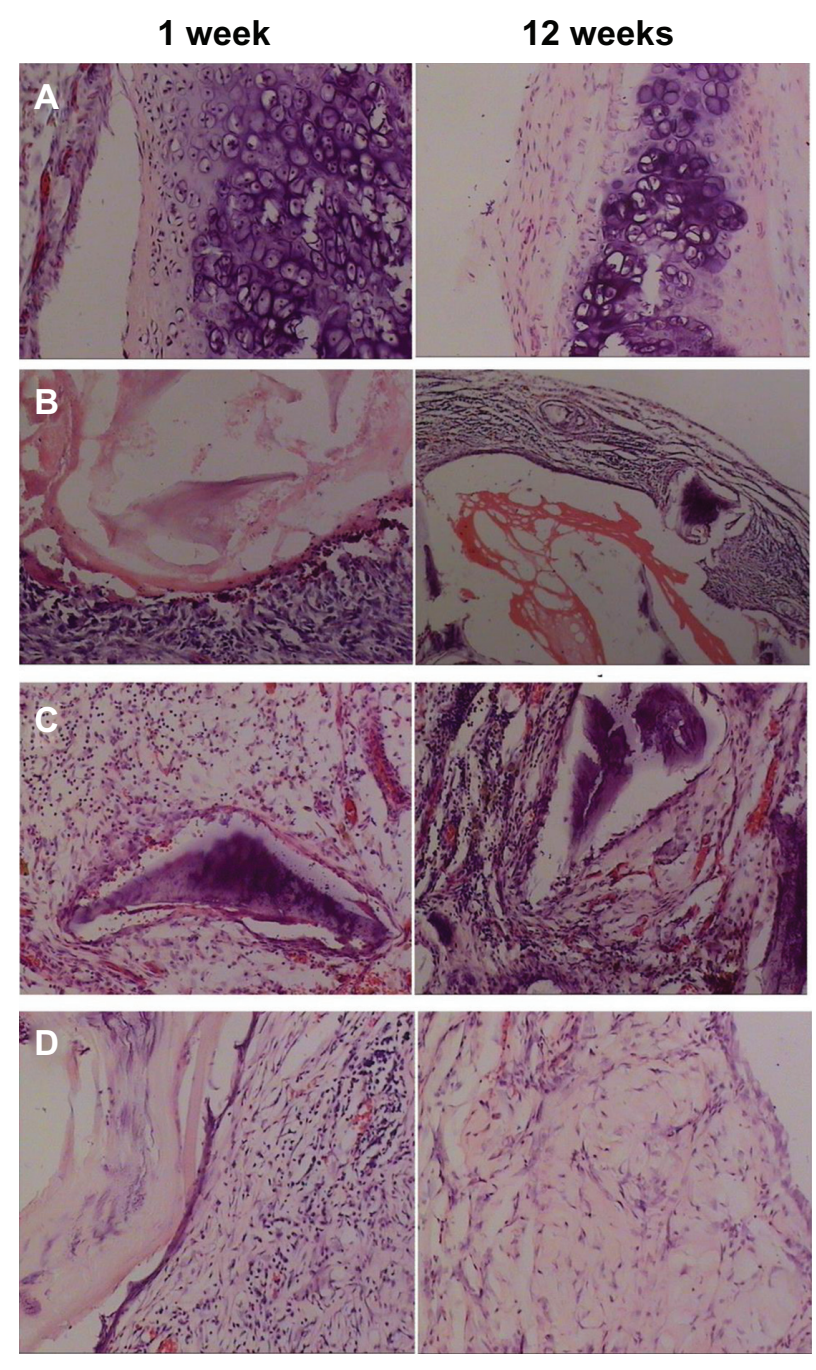

Figure I Hematoxylin and eosin staining showing (A) transplanted group, (B) stent group, (C) non-RNA interference group, and (D) RNA interference group. Note: Magnification I0x. 


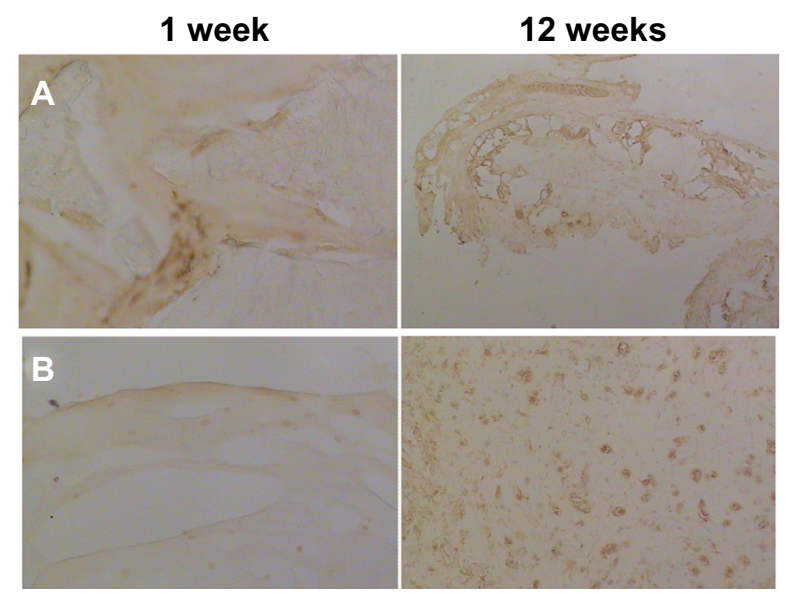

Figure 2 Aggrecan staining showing (A) non-RNA interference group and (B) RNA interference group.

Note: Magnification I0x.

the leukomonocytes showed a dense distribution and were inside the graft (Figure 1).

Aggrecan immunohistochemical staining showed that the positively stained chondrocytes were more significant in the RNAi group, and had positively stained areas around the cells. A higher expression of aggrecan messenger RNA was also detected by RT-PCR in the RNAi group (Figure 2).

The immunological reaction detection found that MHC II-positive cells continued to increase in the normal group, and were also distributed within the graft. However, in the RNAi group they tended to stabilize after 4 weeks and were only distributed in the surrounding part of the graft.

The flow cytometry results showed that the ratio of $\mathrm{CD}^{+} / \mathrm{CD}^{+} \mathrm{T}$-cells of all the transplant groups was nonsignificantly lower than the blank comparison group at 1 week $(P>0.05)$. After 1 week, the ratio of the normal chondrocyte group was always higher than the comparison group at different time points $(P>0.05)$. However, there were no significant differences between the RNAi group, the stent group, and the comparison group $(P>0.05)$ (Table 1).

In addition, the RNAi group showed reduced interferon- $\gamma$ secretion in $\mathrm{CD}^{+} / \mathrm{CD} 8^{+} \mathrm{T}$-cells (Table 2 ).

\section{Discussion}

Cartilage tissue engineering provides a potential efficient therapy for the construction of cartilage defects, which includes three key elements: cell sources, growth factors, and scaffold biomaterials. As a richer source with stable cell characteristics, allogenic chondrocytes have become one main target of seed cells in cartilage tissue engineering research. Clinical and experimental observations have established that transplants of allogenic cartilage usually resist rejection, ${ }^{7}$ while on the other hand, transplants of isolated allogenic chondrocytes evoke a strong reaction leading to cartilage resorption by infiltrating cells. ${ }^{2}$ This particular feature can be explained by the fact that the chondrocytes constitutively express MHC I and possibly possess specific differentiation antigen; ${ }^{8,9}$ however, the chondrocyte's integrity is sequestered by the affluent matrix, thus preventing their contact with the host immunocompetent cells as the matrix does not allow permeability of molecules greater than $60 \mathrm{kDa}^{7}$ The rejection and resorption of cartilage has also been observed in the transplantation of tissue engineering cartilage constructed by allogenic chondrocytes. ${ }^{2}$ Thus, it is important to maintain the quantity of ECM in cartilage allotransplants.

The key roles and early events of aggrecan in the degeneration of cartilage ECM have been reported. ${ }^{5}$ The special enzymes responsible for aggrecan degradation are aggrecanase- 1 and aggrecanase- 2 . This leads to the question of whether the destruction of ECM could be arrested and allograft rejection be weakened or avoided through the inhibition or inactivation of aggrecanase- 1 and aggrecanase- 2 activity. With in vitro experiments, it has been observed that aggrecan and collagen increase significantly

Table I The $\mathrm{CD} 4^{+} / \mathrm{CD} 8^{+}$ratio in different groups

\begin{tabular}{llllll}
\hline $\begin{array}{l}\text { Time point } \\
\text { (weeks) }\end{array}$ & Control group & Transplanted group & Stent group & Non-RNAi group & RNAi group \\
\hline 1 & $1.71 \pm 0.11$ & $1.70 \pm 0.12$ & $1.69 \pm 0.15$ & $1.66 \pm 0.14$ & $1.70 \pm 0.09$ \\
2 & $1.69 \pm 0.12$ & $1.76 \pm 0.21$ & $1.74 \pm 0.07$ & $2.02 \pm 0.14^{*}$ & $1.73 \pm 0.16$ \\
4 & $1.70 \pm 0.08$ & $1.75 \pm 0.15$ & $1.72 \pm 0.22$ & $2.07 \pm 0.12^{*}$ & $1.73 \pm 0.19$ \\
8 & $1.7 \pm 0.16$ & $1.71 \pm 0.08$ & $1.72 \pm 0.15$ & $2.11 \pm 0.23^{*}$ & $1.75 \pm 0.15$ \\
12 & $1.69 \pm 0.13$ & $1.76 \pm 0.31$ & $1.71 \pm 0.17$ & $2.27 \pm 0.26 *$ & $1.77 \pm 0.28$ \\
\hline
\end{tabular}

Note: $* P<0.05$.

Abbreviation: RNAi, RNA interference. 
Table 2 Cytokine expression in $\mathrm{CD}^{+}$and $\mathrm{CD} 8^{+} \mathrm{T}$-cells

\begin{tabular}{|c|c|c|c|c|}
\hline \multirow[t]{2}{*}{ Group } & \multicolumn{2}{|c|}{$\mathrm{CD4}^{+}$T-cells } & \multicolumn{2}{|l|}{$\mathrm{CD8}^{+} \mathrm{T}$-cells } \\
\hline & IFN- $\gamma$ & IL-4 & IFN- $\gamma$ & IL-4 \\
\hline Control group & $6.50+1.42$ & $3.02+0.81$ & $8.10+4.38$ & $2.68+1.12$ \\
\hline Transplanted group & $6.90+1.22$ & $3.62+0.31$ & $8.50+2.12$ & $2.36+0.83$ \\
\hline Stent group & $6.23+0.76$ & $3.96+0.56$ & $8.13+1.86$ & $2.12+0.47$ \\
\hline Non-RNAi group & $8.90+1.79 *$ & $5.24+0.89$ & $10.50+1.17 *$ & $4.46+0.34$ \\
\hline RNAi group & $6.43+0.97$ & $3.4 I+0.54$ & $8.33+3.64$ & $2.79+0.76$ \\
\hline
\end{tabular}

Note: $* p<0.05$.

Abbreviations: $C D$, cluster of differentiation; IFN- $\gamma$, interferon- $\gamma$; IL-4, interleukin 4; RNAi, RNA interference.

after inhibition of aggrecanase- 1 and aggrecanase- 2 by RNAi technology. ${ }^{5}$

\section{Conclusion}

Chondrocytes treated with inhibition of aggrecanase- 1 and aggrecanase- 2 by RNAi could primely construct tissueengineered cartilage in vitro. The chondrocyte phenotypes were maintained, and the amount of aggrecan was upregulated. Grafts showed a high expression of aggrecan and good maintenance of ECM quantity in vivo after allogeneic transplantation.

Immunological rejection exists in transplanted allogenic tissue-engineered cartilage. However, it could be weakened with RNAi-treated chondrocytes. This means that the inhibition of aggrecanase- 1 and aggrecanase- 2 can maintain the barrier structure of ECM, and then effectively preserve the immune tolerance of the transplantation.

\section{Disclosure}

The authors report no conflicts of interest in this work.

\section{References}

1. Temenoff JS, Mikos AG. Review: tissue engineering for regeneration of articular cartilage. Biomaterials. 2000;21(5):431-440.

2. Kawabe N, Yoshinao M. The repair of full-thickness articular cartilage defects. Immune responses to reparative tissue formed by allogeneic growth plate chondrocyte implants. Clin Orthop Relat Res. 1991;(268): 279-293.

3. Hou C, Chang X, Yang Z. [An experimental study on changes in collagen content after homologous cartilage implantation]. Zhonghua Zheng Xing Shao Shang Wai Ke Za Zhi. 1995;11(4):274-276. Chinese.

4. Lohmander LS, Neame PJ, Sandy JD. The structure of aggrecan fragments in human synovial fluid. Evidence that aggrecanase mediates cartilage degradation in inflammatory joint disease, joint injury, and osteoarthritis. Arthritis Rheum. 1993;36(9):1214-1222.

5. Wang ZH, Yang ZQ, He XJ, Wang L, Li LX, Tu JB. Effects of RNAi-mediated inhibition of aggrecanase- 1 and aggrecanase- 2 on rat costochondral chondrocytes in vitro. Acta Pharmacol Sin. 2008;29(10): $1215-1226$.

6. Zhang XF, Zhang F, Liu HY, et al. Celecoxib plays a multiple role to peripheral blood lymphocytes and allografts in acute rejection in rats after cardiac transplantation. Chin Med J (Engl). 2009;122(2):188-192.

7. Langer F, Gross AE. Immunogenicity of allograft articular cartilage. J Bone Joint Surg Am. 1974;56(2):297-304.

8. Langer F, Gross AE, Greaves MF. The auto-immunogenicity of articular cartilage. Clin Exp Immunol. 1972;12(1):31-37.

9. Osiecka-Iwan A, Hyc A, Jozwiak J, Komar A, Niderla J, Moskalewski S. Transplants of rat chondrocytes evoke strong humoral response against chondrocyte-associated antigen in rabbits. Cell Transplant. 2003;12(4):389-398.
Therapeutics and Clinical Risk Management

\section{Publish your work in this journal}

Therapeutics and Clinical Risk Management is an international, peerreviewed journal of clinical therapeutics and risk management, focusing on concise rapid reporting of clinical studies in all therapeutic areas, outcomes, safety, and programs for the effective, safe, and sustained use of medicines. This journal is indexed on PubMed Central, CAS,

\section{Dovepress}

EMBase, Scopus and the Elsevier Bibliographic databases. The manuscript management system is completely online and includes a very quick and fair peer-review system, which is all easy to use. Visit $\mathrm{http}: / /$ www.dovepress.com/testimonials.php to read real quotes from published authors. 